\title{
A note on Razumikhin theorems in uniform ultimate boundedness
}

\author{
Jiemin Zhao
}




\title{
A NOTE ON RAZUMIKHIN THEOREMS IN UNIFORM ULTIMATE BOUNDEDNESS
}

\begin{abstract}
JIEMIN ZHAO
Received 03 May, 2012

Abstract. Uniform boundedness and uniform ultimate boundedness of solutions of retarded functional differential equations are studied by Liapunov functions. The obtained result in this work improves Razumikhin theorems on uniform ultimate boundedness. Some examples are given to illustrate the advantage of the obtained result.
\end{abstract}

2010 Mathematics Subject Classification: 34C11;34K12

Keywords: Razumikhin theorems, solution, boundedness

\section{INTRODUCTION}

Retarded functional differential equations (RFDEs) are a general type of equations and they include ordinary differential equations and differential difference equations [4]. Significance of boundedness (BD) and ultimate boundedness (UB) of partial solutions of RFDEs is widely known. For some problems, one may be interested only in some variables, and it often is very difficult to solve once for all the problems of whole variables about some complex systems. On the other hand, the BD and the UB of whole variables can be obtained by the BD and the UB of partial variables $[4,6,7]$.

In fact, the BD and the UB of partial solutions were studied for ordinary differential equations (ODEs) and difference equations. For some results in the area see, for example, [2,5-7,9-11,13-19] and the references cited therein.

On the results of ODEs, in [5] the authors obtained two Lyapunov-like theorems for the BD and the UB of partial solutions of nonlinear dynamical system

$$
\begin{aligned}
& \dot{x}_{1}(t)=f_{1}\left(x_{1}(t), x_{2}(t)\right), \\
& \dot{x}_{2}(t)=f_{2}\left(x_{1}(t), x_{2}(t)\right),
\end{aligned}
$$

where $x_{1} \in D, D \subseteq \mathbb{R}^{n_{1}}$ is an open set, $x_{2} \in \mathbb{R}^{n_{2}}, f_{1}: D \times \mathbb{R}^{n_{2}} \rightarrow \mathbb{R}^{n_{1}}, f_{2}: D \times$ $\mathbb{R}^{n_{2}} \rightarrow \mathbb{R}^{n_{2}}$ (see Theorem 3.1 and Theorem 3.2). In [6] the author obtained four

This work was supported by China National Natural Science Foundation under Grant No.11171014. 
results (sufficient conditions) for the BD and the UB (dissipation) of partial solutions of the nonlinear autonomous system

$$
\begin{aligned}
\frac{d y}{d t} & =\operatorname{col}\left(\sum_{j=1}^{n} f_{1 j}\left(x_{j}\right), \cdots, \sum_{j=1}^{n} f_{m j}\left(x_{j}\right)\right), \\
\frac{d z}{d t} & =\operatorname{col}\left(\sum_{j=1}^{n} f_{m+1}\left(x_{j}\right), \cdots, \sum_{j=1}^{n} f_{n j}\left(x_{j}\right)\right)
\end{aligned}
$$

(see Theorem 1 - Theorem 4), where $y=\operatorname{col}\left(x_{1}, \cdots, x_{m}\right), z=\operatorname{col}\left(x_{m+1}, \cdots, x_{n}\right)$; $f_{i j}\left(x_{j}\right) \in C(-\infty,+\infty)$. In [7] the authors obtained four results (sufficient conditions) for the uniform ultimate boundedness (uniform dissipation) of partial solutions of the nonlinear nonautonomous system

$$
\begin{aligned}
& \frac{d y}{d t}=\operatorname{col}\left(\sum_{j=1}^{n} f_{1 j}\left(t, x_{j}\right), \cdots, \sum_{j=1}^{n} f_{m j}\left(t, x_{j}\right)\right), \\
& \frac{d z}{d t}=\operatorname{col}\left(\sum_{j=1}^{n} f_{m+1}\left(t, x_{j}\right), \cdots, \sum_{j=1}^{n} f_{n j}\left(t, x_{j}\right)\right)
\end{aligned}
$$

(see Theorem 1 - Theorem 4), where $f_{i j}\left(t, x_{j}\right) \in C\left(\left[t_{0},+\infty\right) \times \mathbb{R}, \mathbb{R}\right)$. In monograph [8] the author obtained some results (sufficient and necessary conditions, and sufficient conditions) for the BD of partial solutions of the nonlinear ordinary differential equation

$$
\dot{x}(t)=f(t, x(t))
$$

(see Theorem 8.38 and Theorem 8.39), where $f \in C\left(I \times \mathbb{R}^{n}, \mathbb{R}^{n}\right)$.

On the results of the BD and the UB of partial solutions of difference equations, in [18] the authors obtained a result (sufficient conditions) for the uniform ultimate boundedness (UUB) of partial solutions of the large-scale discrete system

$$
x_{i}(\tau+1)=f_{i}\left[\tau, x_{i}(\tau)\right]+g_{i}\left[\tau, x_{1}(\tau), \cdots, x_{m}(\tau)\right](i=1,2, \cdots, m),
$$

where $\tau \in I=\left\{\tau_{0}+k: k=0,1,2, \cdots\right\}, x_{i} \in \mathbb{R}^{n_{i}}, f_{i}: I \times \mathbb{R}^{n_{i}} \rightarrow \mathbb{R}^{n_{i}}, g_{i}: I \times \mathbb{R}^{n_{1}} \times$ $\mathbb{R}^{n_{2}} \times \cdots \times \mathbb{R}^{n_{m}} \rightarrow \mathbb{R}^{n_{i}}, \sum_{i=1}^{m} n_{i}=n$ (see Theorem 3). In [15] the authors gave some results (sufficient conditions) for the BD and the UB of partial solutions of discretetime nonlinear dynamical system

$$
\begin{aligned}
& x_{1}(k+1)=f_{1}\left(x_{1}(k), x_{2}(k)\right), \\
& x_{2}(k+1)=f_{2}\left(x_{1}(k), x_{2}(k)\right),
\end{aligned}
$$

where $k \in\{0,1,2, \cdots\}, x_{1} \in D, D \subseteq \mathbb{R}^{n_{1}}$ is an open set, $x_{2} \in \mathbb{R}^{n_{2}}, f_{1}: D \times \mathbb{R}^{n_{2}} \rightarrow$ $\mathbb{R}^{n_{1}}, f_{2}: D \times \mathbb{R}^{n_{2}} \rightarrow \mathbb{R}^{n_{2}}$ (see Theorem 3.2 and Theorem 3.3). In [9] the author 
obtained some results for the BD and the UB of partial solutions of the difference equation

$$
z(\tau+1)=f(\tau, z(\tau))
$$

(see Theorem 1-5), where $z \in \mathbb{R}^{n}, \tau \in I=\{0,1,2, \cdots\}, f: I \times \mathbb{R}^{n} \rightarrow \mathbb{R}^{n}$.

On the $\mathrm{BD}$ and the UB of partial solutions of ODEs and difference equations, one obtained quite a few results. Nonetheless, the results for uniform BD and UUB of partial solutions of RFDEs are still very few [3,4]. In this paper, using Liapunov function (Liapunov functions are simpler than Liapunov functionals), a result of uniform BD and UUB of the partial coordinates or all the coordinates of the solutions of RFDEs is given. Our result improves the well-known Razumikhin theorems on UUB [4]. The result is easier to apply; its application area is widened. Moreover, some examples are given to illustrate the application and advantage of the obtained result at the end.

\section{Preliminaries}

Suppose $r \geq 0$ is a given real number, $\mathbb{R}=(-\infty, \infty), \mathbb{R}^{+}=[0, \infty), \mathbb{R}^{n}$ is an $n$ dimensional linear vector space over the reals with norm $|\cdot|$ (in this paper, if $x \in \mathbb{R}^{n}$ is a column vector, then $|x|$ denotes the Euclidean length of $x:|x|=\left(\sum_{i=1}^{n} x_{i}^{2}\right)^{\frac{1}{2}}$ ),$C=C\left([-r, 0], \mathbb{R}^{n}\right)$ is the Banach space of continuous functions mapping the interval $[-r, 0]$ into $\mathbb{R}^{n}$ with the topology of uniform convergence. We designate the norm of an element $\phi=\left(\phi_{1}, \phi_{2}, \cdots, \phi_{n}\right)^{T}$ in $C$ by $|\phi|=\sup _{-r \leq \theta \leq 0}|\phi(\theta)|$. If

$$
\sigma \in \mathbb{R}, A \geq 0 \quad \text { and } \quad x \in C\left([\sigma-r, \sigma+A], \mathbb{R}^{n}\right),
$$

then for any $t \in[\sigma, \sigma+A]$, we let $x_{t} \in C$ be defined by $x_{t}(\theta)=x(t+\theta), \theta \in[-r, 0]$.

Suppose $f: \mathbb{R} \times C \rightarrow \mathbb{R}^{n}$ is continuous and consider the retarded functional differential equation $[1,4]$

$$
\dot{x}(t)=f\left(t, x_{t}\right) .
$$

We will assume that there is a unique solution $x\left(t, t_{0}, \phi\right)\left(x\left(t_{0}, \phi\right)(t)\right)$ of the Equation (2.1) through $\left(t_{0}, \phi\right) \in \mathbb{R} \times C$. Let

$$
\begin{gathered}
x=\left(x_{1}, x_{2}, \cdots, x_{n}\right)^{T} \in \mathbb{R}^{n}, \phi=\left(\phi_{1}, \phi_{2}, \cdots, \phi_{n}\right)^{T} \in C, \\
x_{i \sim j}=\left(x_{i}, x_{i+1}, \cdots, x_{j}\right)^{T} \in \mathbb{R}^{j+1-i} \quad(1 \leq i \leq j \leq n), \\
x(t)=x\left(t, t_{0}, \phi\right), x_{i \sim j}(t)=x_{i \sim j}\left(t, t_{0}, \phi\right), \\
y(t)=x_{1 \sim m}(t), z(t)=x_{m+1 \sim n}(t) ; \\
C_{i \sim j}=C\left([-r, 0], \mathbb{R}^{j+1-i}\right), \phi_{i \sim j}=\left(\phi_{i}, \phi_{i+1}, \cdots, \phi_{j}\right)^{T} \in C_{i \sim j} .
\end{gathered}
$$


If $V: \mathbb{R} \times \mathbb{R}^{m} \times \mathbb{R}^{n-m} \rightarrow \mathbb{R}$ is a continuous function, then $\dot{V}\left(t, \phi_{1 \sim m}(0), \phi_{m+1 \sim n}(0)\right)$, the derivative of $V$ along the solutions of Equation (2.1) is defined to be

$$
\begin{gathered}
\dot{V}\left(t, \phi_{1 \sim m}(0), \phi_{m+1 \sim n}(0)\right)= \\
\overline{\lim }_{h \rightarrow 0^{+}} \frac{1}{h}\left[V\left(t+h, x_{1 \sim m}(t, \phi)(t+h), x_{m+1 \sim n}(t, \phi)(t+h)\right)-\right. \\
\left.V\left(t, \phi_{1 \sim m}(0), \phi_{m+1 \sim n}(0)\right)\right] .
\end{gathered}
$$

In this paper, suppose that if $\left(x_{1 \sim m}, x_{m+1 \sim n}\right)^{T} \neq 0$, then $V\left(t, x_{1 \sim m}, x_{m+1 \sim n}\right)>0$ for all $t \in \mathbb{R}$.

Definition 1. The partial solutions $x_{1 \sim m}\left(t, t_{0}, \phi\right)(1 \leq m \leq n)$ of the Equation (2.1) are uniformly bounded if, for any $B_{1}>0$, there is a $B_{2}=B_{2}\left(B_{1}\right)>0$ such that, for all $t_{0} \in \mathbb{R}, \phi \in C$, and $|\phi| \leq B_{1}$, we have

$$
\left|x_{1 \sim m}\left(t, t_{0}, \phi\right)\right| \leq B_{2}\left(B_{1}\right)
$$

for all $t \geq t_{0}$.

Definition 2. The partial solutions $x_{1 \sim m}\left(t, t_{0}, \phi\right)$ of the Equation (2.1) are uniformly ultimately bounded if there is a $B>0$ such that, for any $B_{3}>0$, there is a constant $T\left(B_{3}\right)>0$ such that

$$
\left|x_{1 \sim m}\left(t, t_{0}, \phi\right)\right| \leq B
$$

for $t \geq t_{0}+T\left(B_{3}\right)$ for all $t_{0} \in \mathbb{R}, \phi \in C,|\phi| \leq B_{3}$.

\section{MAin RESUlt}

Without loss of generality, we suppose $r>0$. Suppose $f: \mathbb{R} \times C \rightarrow \mathbb{R}^{n}$ takes $\mathbb{R} \times$ (bounded sets of $C$ ) into bounded sets of $\mathbb{R}^{n}$. Suppose $u, v, w, q: \mathbb{R}^{+} \rightarrow \mathbb{R}^{+}$ are continuous, nondecreasing functions, $u(s), v(s), w(s)$ positive for $s>0, u(0)=$ $v(0)=w(0)=q(0)=0, u(s) \rightarrow \infty$ as $s \rightarrow \infty$. Suppose there is a constant $c>0$ such that $\dot{q}(s) \geq c$ for $s \geq 0$.

Theorem 1. Let the following conditions hold.

(1) There are positive integers $m$ and $k$ with $1 \leq m \leq k \leq n$. There is a continuous function $V: \mathbb{R} \times \mathbb{R}^{m} \times \mathbb{R}^{n-m} \rightarrow \mathbb{R}$ such that

$$
u\left(\left|x_{1 \sim m}\right|\right) \leq V\left(t, x_{1 \sim m}, x_{m+1 \sim n}\right) \leq v\left(\left|x_{1 \sim k}\right|\right)
$$

for $t \in \mathbb{R}, x \in \mathbb{R}^{n}$.

(2) There is a continuous, nondecreasing function $p: \mathbb{R}^{+} \rightarrow \mathbb{R}^{+}$with

$$
p(s)>q(s) \text { for } s>0 .
$$


(3) Let $x_{m+1 \sim n} \in \mathbb{R}^{n-m}$ be a vector, and let

$$
x(t)=x_{1 \sim n}(t)=\left(y^{T}(t), z^{T}(t)\right)^{T}=\left(x_{1 \sim m}^{T}(t), x_{m+1 \sim n}^{T}(t)\right)^{T}
$$

be the solution of the Equation (2.1). If there is a constant $H>0$ and if $\left|x_{1 \sim m}(t)\right| \geq H$ for all $t \geq t_{0}$ and

$$
q\left(V\left(s, y(s), x_{m+1 \sim n}\right)\right)<p\left(V\left(t, y(t), x_{m+1 \sim n}\right)\right)
$$

for all $t \geq t_{0}, s \in[t-r, t]$, then

$$
\dot{V}_{(2.1)}(t, y(t), z(t)) \leq-w(|y(t)|)
$$

for all $t \geq t_{0}$.

Under these conditions, the coordinates $x_{1 \sim m}\left(t, t_{0}, \phi\right)$ of the solutions of the Equation (2.1) are uniformly bounded and uniformly ultimately bounded.

Remark 1. The well-known Razumikhin theorems on UUB may be deduced from the above Theorem 1 ( the function $q(s) \equiv s, m=k=n$ ).

\section{Proof of Theorem 1}

In order to prove Theorem 1 in this paper, we need three lemmas.

Although $\left|x_{1 \sim n}(t)\right| \geq H$ is assumed in Lemma 2 and Lemma 3 below, this paper is interested only in the issues of the uniform boundedness and the uniform ultimate boundedness of the partial solutions $x_{1 \sim m}(t)(m \leq n)$. Therefore, condition $\left|x_{1 \sim m}(t)\right| \geq H$ is assumed in Theorem 1. In fact, if $\left|x_{1 \sim m}(t)\right| \geq H$, then $\left|x_{1 \sim n}(t)\right| \geq H\left(\left|x_{1 \sim n}(t)\right| \geq\left|x_{1 \sim m}(t)\right| \geq H\right)$. Consequently, the Theorem 1 in this paper can be proved by Lemma 3 below ( see page 11 ).

Let $\mathbb{R}_{0}=\left[t_{0}, \infty\right)$. If

$$
\bar{V}\left(t, y_{t}, z_{t}\right)=\sup _{\theta \in[-r, 0]} V(t+\theta, y(t+\theta), z(t))
$$

for $(t, x(t+\theta)) \in \mathbb{R}_{0} \times C, \theta \in[-r, 0]\left(x(t+\theta)=\left(y_{t}^{T}, z_{t}^{T}\right)^{T} \in C\right)$, then there is a $\theta_{0}=\theta_{0}(t)$ in $[-r, 0]$ such that

$$
\bar{V}\left(t, y_{t}, z_{t}\right)=V\left(t+\theta_{0}(t), y\left(t+\theta_{0}(t)\right), z(t)\right)
$$

and either $\theta_{0}(t)=0$ or $\theta_{0}(t)<0$ and

$$
V(t+\theta, y(t+\theta), z(t))<V\left(t+\theta_{0}(t), y\left(t+\theta_{0}(t)\right), z(t)\right)
$$

if $\theta_{0}(t)<\theta \leq 0$. 
Lemma 1. Let $\left(y^{T}(t), z^{T}(t)\right)^{T}$ be the solution of Equation (2.1). If $\theta_{0}=\theta_{0}(t)<0$ for some $t \in \mathbb{R}_{0}$, then

$$
\dot{\bar{V}}\left(t, y_{t}, z_{t}\right)=0
$$

Proof. If

$$
V(t+h+\theta, y(t+h+\theta), z(t+h))>V\left(t+\theta_{0}, y\left(t+\theta_{0}\right), z(t)\right)
$$

for $\theta \in\left(\theta_{0}, 0\right]$, and sufficiently small $h>0$, then we would have

$$
\lim _{h \rightarrow 0^{+}} V(t+h+\theta, y(t+h+\theta), z(t+h)) \geq \lim _{h \rightarrow 0^{+}} V\left(t+\theta_{0}, y\left(t+\theta_{0}\right), z(t)\right),
$$

and using the facts that $V, y(t+h+\theta)$, and $z(t+h)$ are continuous, we get

$$
V(t+\theta, y(t+\theta), z(t)) \geq V\left(t+\theta_{0}, y\left(t+\theta_{0}\right), z(t)\right)
$$

for $\theta \in\left(\theta_{0}, 0\right]$, which contradicts the (4.3). So

$$
V(t+h+\theta, y(t+h+\theta), z(t+h)) \leq V\left(t+\theta_{0}, y\left(t+\theta_{0}\right), z(t)\right)
$$

for $\theta \in\left(\theta_{0}, 0\right]$, and sufficiently small $h>0$. From this, we have

$$
\bar{V}\left(t+h, y_{t+h}, z_{t+h}\right)=\bar{V}\left(t, y_{t}, z_{t}\right)
$$

for sufficiently small $h>0$ [4]. Therefore, $\dot{\bar{V}}\left(t, y_{t}, z_{t}\right)=0$. The proof of the Lemma 1 is therefore complete.

Lemma 2. Let the following conditions hold.

(1) There is a continuous function $V: \mathbb{R} \times \mathbb{R}^{m} \times \mathbb{R}^{n-m} \rightarrow \mathbb{R}$ such that

$$
u\left(\left|x_{1 \sim m}\right|\right) \leq V\left(t, x_{1 \sim m}, x_{m+1 \sim n}\right) \leq v\left(\left|x_{1 \sim k}\right|\right) \quad(1 \leq m \leq k \leq n)
$$
for $t \in \mathbb{R}, x \in \mathbb{R}^{n}$.

(2) Let $x(t)=x_{1 \sim n}(t)=\left(y^{T}(t), z^{T}(t)\right)^{T}=\left(x_{1 \sim m}^{T}(t), x_{m+1 \sim n}^{T}(t)\right)^{T}$ be the solution of the Equation (2.1). If $\left|x_{1 \sim n}(t)\right| \geq H$ for all $t \geq t_{0}$ and

$$
V(t+\theta, y(t+\theta), z(t)) \leq V(t, y(t), z(t))
$$

for all $t \geq t_{0}, \theta \in[-r, 0]$, then

$$
\dot{V}_{(2.1)}(t, y(t), z(t)) \leq 0
$$

for all $t \geq t_{0}$.

Under these conditions, the coordinates $x_{1 \sim m}\left(t, t_{0}, \phi\right)$ of the solutions of the Equation (2.1) are uniformly bounded .

Proof. Let $B_{1} \geq H$ be given, $t_{0} \in \mathbb{R}, \phi \in C$, and $|\phi| \leq B_{1}$. Choose $B_{2}>B_{1}$ such that $u\left(B_{2}\right) \geq v\left(B_{1}\right)$. Consider any solution $x(t)$ of (2.1). Using (4.2), (4.7) and our choice of $u\left(B_{2}\right)$, we get

$$
\begin{aligned}
& \bar{V}\left(t_{0}, y_{t_{0}}, z_{t_{0}}\right)=V\left(t_{0}+\theta_{0}, y\left(t_{0}+\theta_{0}\right), z\left(t_{0}\right)\right) \leq \\
& v\left(\left|\left(y^{T}\left(t_{0}+\theta_{0}\right), x_{m+1 \sim k}^{T}\left(t_{0}\right)\right)^{T}\right|\right) \leq v(|\phi|) \leq v\left(B_{1}\right) \leq u\left(B_{2}\right) .
\end{aligned}
$$


We claim that

$$
\bar{V}\left(t, y_{t}, z_{t}\right) \leq u\left(B_{2}\right) \text { for all } t \geq t_{0} .
$$

If this were not so, then there would exist a $t^{*}, t^{*}>t_{0}$, such that

$$
\bar{V}\left(t^{*}, y_{t^{*}}, z_{t^{*}}\right)>u\left(B_{2}\right) \text {. }
$$

Since (4.9), (4.11) and $\bar{V}\left(t, y_{t}, z_{t}\right)$ is continuous in t, there exists $t_{1} \in\left[t_{0}, t^{*}\right]$ such that

$$
\bar{V}\left(t_{1}, y_{t_{1}}, z_{t_{1}}\right)=u\left(B_{2}\right)<\bar{V}\left(t^{*}, y_{t^{*}}, z_{t^{*}}\right) .
$$

Therefore the mean value theorem yields there exists $\bar{t} \in\left[t_{1}, t^{*}\right]$ such that

$$
\dot{\bar{V}}\left(\bar{t}, y_{\bar{t}}, z_{\bar{t}}\right)>0 \text {. }
$$

If $\theta_{0}<0$, then using Lemma 1 , we have $\dot{\bar{V}}\left(\bar{t}, y_{\bar{t}}, z_{\bar{t}}\right)=0$, which contradicts the (4.13). If $\theta_{0}=0$, then using (4.2), (4.7), (4.12), and our choice of $u\left(B_{2}\right)$, we have

$$
v\left(B_{1}\right) \leq u\left(B_{2}\right) \leq \bar{V}\left(\bar{t}, y_{\bar{t}}, z_{\bar{t}}\right)=V(\bar{t}, y(\bar{t}), z(\bar{t})) \leq v\left(\left|x_{1 \sim k}(\bar{t})\right|\right) \leq v(|x(\bar{t})|) .
$$

Using the fact that $v$ is nondecreasing, we get $B_{1} \leq|x(\bar{t})| \quad\left(|x(\bar{t})| \geq B_{1} \geq H\right)$. If $\theta_{0}=0$, then using (4.1) and (4.2), we have

$$
\begin{aligned}
& V(\bar{t}+\theta, y(\bar{t}+\theta), z(\bar{t})) \leq \sup _{\theta \in[-r, 0]} V(\bar{t}+\theta, y(\bar{t}+\theta), z(\bar{t}))= \\
& \bar{V}\left(\bar{t}, y_{\bar{t}}, z_{\bar{t}}\right)=V\left(\bar{t}+\theta_{0}, y\left(\bar{t}+\theta_{0}\right), z(\bar{t})\right)=V(\bar{t}, y(\bar{t}), z(\bar{t}))
\end{aligned}
$$

for $\theta \in[-r, 0], \bar{t} \geq t_{0}$. From (4.8), (4.14) and $|x(\bar{t})| \geq H$, we have $\dot{\bar{V}}\left(\bar{t}, y_{\bar{t}}, z_{\bar{t}}\right)=$ $\dot{V}\left(\bar{t}, y_{\bar{t}}, z_{\bar{t}}\right) \leq 0$, which contradicts the (4.13). So $\bar{V}\left(t, y_{t}, z_{t}\right) \leq u\left(B_{2}\right)$ for all $t \geq t_{0}$.

From (4.1), (4.7) and (4.10), we obtain

$$
\begin{aligned}
& u(|y(t)|) \leq V(t, y(t), z(t))= \\
& V(t+0, y(t+0), z(t)) \leq \sup _{\theta \in[-r, 0]} V(t+\theta, y(t+\theta), z(t))= \\
& \bar{V}\left(t, y_{t}, z_{t}\right) \leq u\left(B_{2}\right)
\end{aligned}
$$

for all $t \geq t_{0}$. Using the fact that $u$ is nondecreasing, we get $|y(t)| \leq B_{2}$ for all $t \geq t_{0}$. The proof of the Lemma 2 is therefore complete.

Lemma 3. Let the following conditions hold.

(1) There are positive integers $m$ and $k$ with $1 \leq m \leq k \leq n$. There is a continuous function $V: \mathbb{R} \times \mathbb{R}^{m} \times \mathbb{R}^{n-m} \rightarrow \mathbb{R}$ such that

$$
u\left(\left|x_{1 \sim m}\right|\right) \leq V\left(t, x_{1 \sim m}, x_{m+1 \sim n}\right) \leq v\left(\left|x_{1 \sim k}\right|\right)
$$

for $t \in \mathbb{R}, x \in \mathbb{R}^{n}$.

(2) There is a continuous, nondecreasing function $p: \mathbb{R}^{+} \rightarrow \mathbb{R}^{+}$with

$$
p(s)>q(s) \text { for } s>0 .
$$


(3) Let $x(t)=x_{1 \sim n}(t)=\left(y^{T}(t), z^{T}(t)\right)^{T}=\left(x_{1 \sim m}^{T}(t), x_{m+1 \sim n}^{T}(t)\right)^{T}$ be the solution of the Equation (2.1). If $\left|x_{1 \sim n}(t)\right| \geq H$ for all $t \geq t_{0}$ and

$$
q(V(t+\theta, y(t+\theta), z(t)))<p(V(t, y(t), z(t)))
$$

for all $t \geq t_{0}, \theta \in[-r, 0]$, then

$$
\dot{V}_{(2.1)}(t, y(t), z(t)) \leq-w(H)
$$

for all $t \geq t_{0}$.

Under these conditions, the coordinates $x_{1 \sim m}\left(t, t_{0}, \phi\right)$ of the solutions of the Equation (2.1) are uniformly bounded and uniformly ultimately bounded.

Proof. First, we show the uniform boundedness.

If $|x(t)| \geq H$ and $V(t+\theta, y(t+\theta), z(t)) \leq V(t, y(t), z(t))$ for $\theta \in[-r, 0], t \geq t_{0}$, then using (4.16) and the fact that $q$ is nondecreasing, we have

$$
q(V(t+\theta, y(t+\theta), z(t))) \leq q(V(t, y(t), z(t)))<p(V(t, y(t), z(t)))
$$

for $\theta \in[-r, 0], t \geq t_{0}$; this follows since $V(t, y(t), z(t))>0 \quad(|x(t)| \geq H>0)$. Hypothesis (4.17) implies $\dot{V}(t, y(t), z(t)) \leq-w(H) \leq 0$. Lemma 2 implies the coordinates $x_{1 \sim m}\left(t, t_{0}, \phi\right)$ of the solutions of the Equation (2.1) are uniformly bounded.

We now show the uniform ultimate boundedness. Choose $B>H$ such that $u(B)>$ $v(H)$. Let $B_{3} \geq H$ be given. The same argument as in the proof of Lemma 2 shows there is a $B_{4}>B$ such that for any $t_{0} \in \mathbb{R}, t \geq t_{0}$, and $\phi \in C$ with $|\phi| \leq B_{3}$, the $B_{4}$ and $u\left(B_{4}\right)$ satisfy

$$
\begin{aligned}
& u(B)<u\left(B_{4}\right), \bar{V}\left(t, y_{t}, z_{t}\right) \leq u\left(B_{4}\right) \text { and } \\
& |y(t)| \leq B_{4} \text { for all } t \geq t_{0}(\text { see }(4.10)) .
\end{aligned}
$$

If $\tilde{x}=\left(B_{4}, 0, \cdots, 0\right)^{T} \in \mathbb{R}^{n}$, then using (4.15) and (4.19), we have

$$
\begin{aligned}
& 0<u(B)<u\left(B_{4}\right)=u\left(\left|\tilde{x}_{1 \sim m}\right|\right) \leq \\
& V\left(t, \tilde{x}_{1 \sim m}, \tilde{x}_{m+1 \sim n}\right) \leq v\left(\left|\tilde{x}_{1 \sim k}\right|\right)=v\left(B_{4}\right) .
\end{aligned}
$$

From the properties of the functions $p(s)$ and $q(s)$, there is a number $a>0$ such that

$$
p(s)-q(s)>a \text { for } u(B) \leq s \leq v\left(B_{4}\right) .
$$

Let $N$ be the first nonnegative integer such that

$$
q(u(B))+N a \geq q\left(v\left(B_{4}\right)\right),
$$

and let

$$
T_{l}=\frac{l q\left(v\left(B_{4}\right)\right)}{c w(H)} \quad(l=0,1,2, \cdots, N) .
$$

First, we show that

$$
q(V(t, y(t), z(t))) \leq q(u(B))+(N-l) a
$$


for all $t \geq t_{0}+l r+T_{l}, l=0,1,2, \cdots, N$. From (4.19)and (4.20), we have

$$
V(t, y(t), z(t)) \leq \bar{V}\left(t, y_{t}, z_{t}\right) \leq u\left(B_{4}\right) \leq v\left(B_{4}\right) \text { for } t \geq t_{0} .
$$

If $l=0$, then using (4.22) and (4.25), we have

$$
\begin{aligned}
& q(V(t, y(t), z(t))) \leq q\left(\bar{V}\left(t, y_{t}, z_{t}\right)\right) \leq q\left(v\left(B_{4}\right)\right) \leq \\
& q(u(B))+N a=q(u(B))+(N-0) a
\end{aligned}
$$

for all $t \geq t_{0}=t_{0}+0 \cdot r+T_{0}$. That is, the inequality (4.24) is true for the $l=0$.

We now show that inequality (4.24) is true for $l=1$. If

$$
q(u(B))+(N-1) a<q\left(V\left(\tilde{t}_{0}, y\left(\tilde{t}_{0}\right), z\left(\tilde{t}_{0}\right)\right)\right)
$$

for $\tilde{\mathfrak{t}}_{0} \geq t_{0}$, then, since $q(u(B)) \leq q(u(B))+(N-1) a<q\left(V\left(\tilde{t}_{0}, y\left(\tilde{t}_{0}\right), z\left(\tilde{t}_{0}\right)\right)\right)$, it follows that

$$
u(B)<V\left(\tilde{t}_{0}, y\left(\tilde{t}_{0}\right), z\left(\tilde{t}_{0}\right)\right) .
$$

From (4.25) and (4.27), we obtain

$$
u(B)<V\left(\tilde{t}_{0}, y\left(\tilde{t}_{0}\right), z\left(\tilde{t}_{0}\right)\right) \leq v\left(B_{4}\right) .
$$

Using (4.15), (4.27), and our choice of $v(H)$, we have

$$
v(H)<u(B)<V\left(\tilde{t}_{0}, y\left(\tilde{t}_{0}\right), z\left(\tilde{t}_{0}\right)\right) \leq v\left(\left|x_{1 \sim k}\left(\tilde{t}_{0}\right)\right|\right) \leq v\left(\left|x\left(\tilde{t}_{0}\right)\right|\right),
$$

and using the fact that $v$ is nondecreasing, we get $H \leq\left|x\left(\tilde{t}_{0}\right)\right|$. Using (4.21), (4.22), (4.25), (4.26) and (4.28), we get

$$
\begin{aligned}
& p\left(V\left(\tilde{t}_{0}, y\left(\tilde{t}_{0}\right), z\left(\tilde{t}_{0}\right)\right)\right)>q\left(V\left(\tilde{t}_{0}, y\left(\tilde{t}_{0}\right), z\left(\tilde{t}_{0}\right)\right)\right)+a> \\
& q(u(B))+(N-1) a+a=q(u(B))+N a \geq q\left(v\left(B_{4}\right)\right) \geq \\
& q\left(\bar{V}\left(\tilde{t}_{0}, y_{\tilde{t}_{0}}, z_{\tilde{t}_{0}}\right) \geq q\left(V\left(\tilde{t}_{0}+\theta, y\left(\tilde{t}_{0}+\theta\right), z\left(\tilde{t}_{0}\right)\right)\right)\right.
\end{aligned}
$$

for $\theta$ in $[-r, 0]$. Hypothesis (4.17) implies

$$
\dot{V}\left(\tilde{t}_{0}, y\left(\tilde{t}_{0}\right), z\left(\tilde{t}_{0}\right)\right) \leq-w(H)<0
$$

for $\tilde{t}_{0} \geq t_{0}$. Therefore, if there is a $t_{0}^{*} \in\left[t_{0}, t_{0}+T_{1}\right)$ such that

$$
q\left(V\left(t_{0}^{*}, y\left(t_{0}^{*}\right), z\left(t_{0}^{*}\right)\right)\right) \leq q(u(B))+(N-1) a,
$$

then using (4.26) and (4.31), we have

$$
q(V(t, y(t), z(t))) \leq q(u(B))+(N-1) a
$$

for $t \geq t_{0}+r+T_{1}$. If

$$
q(u(B))+(N-1) a<q(V(t, y(t), z(t)))
$$

for all $t \in\left[t_{0}, t_{0}+T_{1}\right)$, then using (4.23) and (4.31), we have

$$
\begin{aligned}
& q\left(V\left(t_{0}+T_{1}, y\left(t_{0}+T_{1}\right), z\left(t_{0}+T_{1}\right)\right)\right)= \\
& q\left(V\left(t_{0}, y\left(t_{0}\right), z\left(t_{0}\right)\right)\right)+\dot{q}(V(\xi, y(\xi), z(\xi))) \dot{V}(\xi, y(\xi), z(\xi)) T_{1}=
\end{aligned}
$$




$$
\begin{aligned}
& q\left(V\left(t_{0}, y\left(t_{0}\right), z\left(t_{0}\right)\right)\right)+\dot{q}(V(\xi, y(\xi), z(\xi))) \dot{V}(\xi, y(\xi), z(\xi)) \frac{q\left(v\left(B_{4}\right)\right)}{c w(H)} \leq \\
& q\left(V\left(t_{0}, y\left(t_{0}\right), z\left(t_{0}\right)\right)\right)-\dot{q}(V(\xi, y(\xi), z(\xi))) \frac{q\left(v\left(B_{4}\right)\right) w(H)}{c w(H)}= \\
& q\left(V\left(t_{0}, y\left(t_{0}\right), z\left(t_{0}\right)\right)\right)-\dot{q}(V(\xi, y(\xi), z(\xi))) \frac{q\left(v\left(B_{4}\right)\right)}{c} \leq \\
& q\left(V\left(t_{0}, y\left(t_{0}\right), z\left(t_{0}\right)\right)\right)-c \frac{q\left(v\left(B_{4}\right)\right)}{c}= \\
& q\left(V\left(t_{0}, y\left(t_{0}\right), z\left(t_{0}\right)\right)\right)-q\left(v\left(B_{4}\right)\right) \leq q\left(v\left(B_{4}\right)\right)-q\left(v\left(B_{4}\right)\right)=0 \leq \\
& q(u(B))+(N-1) a .
\end{aligned}
$$

Therefore, we have

$$
q(V(t, y(t), z(t))) \leq q(u(B))+(N-1) a \text { for all } t \geq t_{0}+r+T_{1} .
$$

Thus, the inequality (4.24) is true for the $l=1$.

The arguments are essentially the same as before, we are able to show that

$$
q(V(t, y(t), z(t))) \leq q(u(B))+(N-K) a
$$

for all $t \geq t_{0}+K r+T_{K}, K=2,3, \cdots, N[4,12]$. Therefore, we have

$$
q(V(t, y(t), z(t))) \leq q(u(B))+(N-l) a
$$

for all $t \geq t_{0}+l r+T_{l}, l=0,1,2, \cdots, N$. For $l=N$, we have

$$
q(V(t, y(t), z(t))) \leq q(u(B))+(N-N) a=q(u(B))
$$

for all $t \geq t_{0}+N r+T_{N}$. Hypothesis (4.15) implies

$$
q(u(|y(t)|)) \leq q(V(t, y(t), z(t))) \leq q(u(B))
$$

for all $t \geq t_{0}+N r+T_{N}$ and hence

$$
\left|\left(x_{1}\left(t, t_{0}, \phi\right), x_{2}\left(t, t_{0}, \phi\right), \cdots, x_{m}\left(t, t_{0}, \phi\right)\right)^{T}\right| \leq B
$$

for all $t \geq t_{0}+N r+T_{N}$. Let $T=N r+T_{N}$. Thus, this completes the proof of uniform ultimate boundedness. The proof of the Lemma 3 is therefore complete.

Now we are in the position to prove our Theorem 1.

Proof of Theorem 1. If

$$
\left|x_{1 \sim m}(t)\right| \geq H
$$

for all $t \geq t_{0}$ and

$$
q\left(V\left(s, y(s), x_{m+1 \sim n}\right)\right)<p\left(V\left(t, y(t), x_{m+1 \sim n}\right)\right)
$$

for all $t \geq t_{0}, s \in[t-r, t], x_{m+1 \sim n} \in \mathbb{R}^{n-m}$, then using (c), we have

$$
\dot{V}_{(2.1)}(t, y(t), z(t)) \leq-w(|y(t)|)
$$


for all $t \geq t_{0}$. From (4.34) and (4.35), we obtain

$$
\left|x_{1 \sim n}(t)\right| \geq\left|x_{1 \sim m}(t)\right| \geq H
$$

for all $t \geq t_{0}$ and

$$
q(V(t+\theta, y(t+\theta), z(t)))<p(V(t, y(t), z(t)))
$$

for all $t \geq t_{0}, \theta \in[-r, 0]$. Using (4.36), (4.37), and the fact that $w$ is nondecreasing, we get

$$
\dot{V}_{(2.1)}(t, y(t), z(t)) \leq-w(|y(t)|)=-w\left(\left|x_{1 \sim m}(t)\right|\right) \leq-w(H)
$$

for all $t \geq t_{0}$. Lemma 3 implies the coordinates $x_{1 \sim m}\left(t, t_{0}, \phi\right)$ of the solutions of the Equation (2.1) are uniformly bounded and uniformly ultimately bounded (see the (4.37), the (4.38), and the (4.39)). The proof of Theorem 1 is therefore complete.

\section{EXAMPLES}

We give the following examples in order to illustrate the application and advantage of the Theorem 1 in this paper [4,20,21]. It is easy to see that the well-known Razumikhin theorems on uniform ultimate boundedness cannot apply to the following examples.

Example 1. Consider the scalar equation

$$
\begin{aligned}
\dot{x}(t)= & -\sum_{j=1}^{n} b_{j}(t) x^{2 k-1}(t)\left[\alpha \arctan \beta x^{2 m}\left(t-\tau_{j}(t)\right)+\gamma x^{2 m}\left(t-\tau_{j}(t)\right)\right] \\
& -a(t) x^{2 k-1}(t)\left[\alpha \arctan \beta x^{2 m}(t)+\gamma x^{2 m}(t)\right]+\tilde{p}(t),
\end{aligned}
$$

where $\tilde{p}(t), a(t), b_{j}(t)$, and $\tau_{j}(t)$ are bounded continuous functions on $\mathbb{R}, \alpha, \beta, \gamma=$ const., $\alpha \beta>0, \gamma>0, k=1,2, \cdots, K, m=1,2, \cdots, M$.

We make the following assumptions on Equation (5.1): there is a $q_{0} \in(0,1)$ such that

$$
a(t) \geq \delta>0, \sum_{j=1}^{n}\left|b_{j}(t)\right| \leq \delta q_{0}, 0 \leq \tau_{j}(t) \leq r \quad(j=1,2, \cdots, n)
$$

for all $t \in \mathbb{R}, q_{0}, \delta, r=$ const.

Under the above hypotheses, we will show that the solutions of the Equation (5.1) are uniformly bounded and uniformly ultimately bounded. 
In fact, since $q_{0} \in(0,1)$, there is a $q_{1}>1$ such that $q_{0} q_{1}<1$. If

$$
\begin{aligned}
& p(s)=q_{1} q(s), q(s)=\alpha \arctan \beta s+\gamma s, V(x)=x^{2 m}, \\
& |x(t)| \geq H=\text { const. }>0, \text { and } \\
& p(V(x(t)))>q(V(x(s))), s \in[t-r, t]\left(\Rightarrow q _ { 1 } \left(\alpha \arctan \beta x^{2 m}(t)+\right.\right. \\
& \left.\left.\gamma x^{2 m}(t)\right)>\alpha \arctan \beta x^{2 m}\left(t-\tau_{j}(t)\right)+\gamma x^{2 m}\left(t-\tau_{j}(t)\right), j=1,2, \cdots, n\right),
\end{aligned}
$$

then

$$
\begin{gathered}
\dot{V}_{(5.1)}(x(t))=2 m x^{2(k+m-1)}(t)\left\{-a(t)\left[\alpha \arctan \beta x^{2 m}(t)+\gamma x^{2 m}(t)\right]\right. \\
\left.-\sum_{j=1}^{n} b_{j}(t)\left[\alpha \arctan \beta x^{2 m}\left(t-\tau_{j}(t)\right)+\gamma x^{2 m}\left(t-\tau_{j}(t)\right)\right]\right\} \\
+2 m \tilde{p}(t) x^{2 m-1}(t) \\
\leq 2 m x^{2(k+m-1)}(t)\left\{-a(t)\left[\alpha \arctan \beta x^{2 m}(t)+\gamma x^{2 m}(t)\right]\right. \\
\left.+\sum_{j=1}^{n}\left|b_{j}(t)\right| \cdot q_{1}\left[\alpha \arctan \beta x^{2 m}(t)+\gamma x^{2 m}(t)\right]\right\}+2 m \tilde{p}(t) x^{2 m-1}(t) \\
=2 m x^{2(k+m-1)}(t)\left[\alpha \arctan \beta x^{2 m}(t)+\gamma x^{2 m}(t)\right] \times \\
{\left[-a(t)+q_{1} \sum_{j=1}^{n}\left|b_{j}(t)\right|\right]+2 m \tilde{p}(t) x^{2 m-1}(t)} \\
\leq 2 m x^{2(k+m-1)}(t)\left[\alpha \arctan \beta x^{2 m}(t)+\gamma x^{2 m}(t)\right] \times \\
{\left[-\delta+q_{0} q_{1} \delta\right]+2 m \tilde{p}(t) x^{2 m-1}(t)} \\
\leq-2 m \delta\left(1-q_{0} q_{1}\right) x^{2(k+m-1)}(t)\left[\alpha \arctan \beta x^{2 m}(t)+\gamma x^{2 m}(t)\right] \\
+2 m \tilde{p}(t) x^{2 m-1}(t) .
\end{gathered}
$$

By choosing $H_{1} \geq H=$ const $>0$ appropriately ( $\tilde{p}(t)$ is bounded continuous function on $\mathbb{R}$, constant $\left.\delta\left(1-q_{0} q_{1}\right)>0, \alpha \beta>0, \gamma>0\right)$, we obtain a positive constant $\mu$ such that

$$
2 m\left\{\delta\left(1-q_{0} q_{1}\right)-\frac{\tilde{p}(t)}{x^{2 k-1}(t)\left[\alpha \arctan \beta x^{2 m}(t)+\gamma x^{2 m}(t)\right]}\right\} \geq \mu
$$

for $|x(t)| \geq H_{1}$ and $p(V(x(t)))>q(V(\phi(\theta))), \theta \in[-r, 0]$. From this, we have

$$
\begin{aligned}
\dot{V}_{(5.1)}(x(t)) & \leq-2 m \delta\left(1-q_{0} q_{1}\right) x^{2(k+m-1)}(t)\left[\alpha \arctan \beta x^{2 m}(t)+\gamma x^{2 m}(t)\right] \\
& +2 m \tilde{p}(t) x^{2 m-1}(t)
\end{aligned}
$$




$$
\begin{aligned}
& =-2 m\left\{\delta\left(1-q_{0} q_{1}\right)-\frac{\tilde{p}(t)}{x^{2 k-1}(t)\left[\alpha \arctan \beta x^{2 m}(t)+\gamma x^{2 m}(t)\right]}\right\} \\
& \times x^{2(k+m-1)}(t)\left[\alpha \arctan \beta x^{2 m}(t)+\gamma x^{2 m}(t)\right] \\
& \leq-\mu x^{2(k+m-1)}(t)\left[\alpha \arctan \beta x^{2 m}(t)+\gamma x^{2 m}(t)\right]
\end{aligned}
$$

for $|x(t)| \geq H_{1}$ and $p(V(x(t)))>q(V(\phi(\theta))), \theta \in[-r, 0]$. Therefore, the Theorem 1 implies the solutions of the Equation (5.1) are uniformly bounded and uniformly ultimately bounded.

Example 2. Consider the second-order system

$$
\begin{aligned}
\dot{x}(t)= & 2 y(t), \\
\dot{y}(t) & =-f(x(t))-\Phi(t, y(t)) a^{y^{2}(t)} \\
& +\tilde{p}(t)+y(t) \int_{-r}^{0} g(x(t+\theta)) a^{y^{2}(t+\theta)} d \theta,
\end{aligned}
$$

where $a=$ constant $>1$.

We make the following assumptions on System (5.2):

(a) $\Phi: \mathbb{R}^{2} \rightarrow \mathbb{R}$ is continuous, $\Phi$ takes $\mathbb{R} \times$ (bounded sets of $\mathbb{R}$ ) into bounded sets and there are constants $a_{0}>0, H>0$, such that

$$
\frac{\Phi(t, y)}{y}>a_{0}>0 \text { for } t \in \mathbb{R},|y| \geq H .
$$

(b) $f: \mathbb{R} \rightarrow \mathbb{R}$ is continuous and $f(x) \operatorname{sgn} x \rightarrow \infty$ as $|x| \rightarrow \infty$.

(c) $\tilde{p}: \mathbb{R} \rightarrow \mathbb{R}$ is bounded and continuous.

(d) $g: \mathbb{R} \rightarrow \mathbb{R}$ is continuous and there is a constant $L>0$, such that

$$
|g(x)| \leq L \text { for all } x \in \mathbb{R} \text {. }
$$

(e) $\operatorname{Lr}<a_{0}$.

It is always assumed that a uniqueness result holds for the solutions of System (5.2). Under the above hypotheses, we will show that the second coordinate of the solutions of the System (5.2) is uniformly bounded and uniformly ultimately bounded. 
In fact, since $L r<a_{0}$, there is a $q_{1}>1$ such that $q_{1} L r<a_{0}$. If

$$
\begin{aligned}
& q(s)=a^{s}-1, p(s)=q_{1} q(s), V(x, y)=F(x)+y^{2}, F(x)=\int_{0}^{x} f(s) d s, \\
& |y(t)| \geq H, \text { and } \\
& q(V(x, y(s)))<p(V(x, y(t))), s \in[t-r, t](q(V(x, y(t+\theta)))< \\
& p(V(x, y(t))), \theta \in[-r, 0]) \\
& \left(\Rightarrow a^{F(x)+y^{2}(t+\theta)}-1<q_{1}\left(a^{F(x)+y^{2}(t)}-1\right)\right. \\
& \Rightarrow a^{F(x)+y^{2}(t+\theta)}<a^{F(x)+y^{2}(t+\theta)}+\left(q_{1}-1\right)<q_{1} a^{F(x)+y^{2}(t)} \\
& \left.\Rightarrow a^{y^{2}(t+\theta)}<q_{1} a^{y^{2}(t)}\right),
\end{aligned}
$$

then

$$
\begin{aligned}
\dot{V}_{(5.2)}(x(t), y(t))= & 2 y(t)\left[-\Phi(t, y(t)) a^{y^{2}(t)}+\tilde{p}(t)+\right. \\
& \left.y(t) \int_{-r}^{0} g(x(t+\theta)) a^{y^{2}(t+\theta)} d \theta\right] \\
\leq & \left.2 y^{2}(t)\left[-a_{0} a^{y^{2}(t)}+\int_{-r}^{0}|g(x(t+\theta))| a^{y^{2}(t+\theta)} d \theta\right)\right] \\
& +2|y(t)||\tilde{p}(t)| \\
\leq & -2\left(a_{0}-q_{1} L r\right) y^{2}(t) a^{y^{2}(t)}+2|y(t)||\tilde{p}(t)|,
\end{aligned}
$$

By choosing $H_{1} \geq H$ appropriately ( $\tilde{p}(t)$ is bounded continuous function, constant $\left.\left(a_{0}-q_{1} L r\right)>0\right)$, we obtain a positive constant $\mu$ such that

$$
2\left[\left(a_{0}-q_{1} L r\right)-\frac{|\tilde{p}(t)|}{|y(t)| a^{y^{2}(t)}}\right] \geq \mu
$$

for $|y(t)| \geq H_{1}$ and $q(V(x, y(s)))<p(V(x, y(t))), s \in[t-r, t]$. From this, we have

$$
\begin{aligned}
\dot{V}_{(5.2)}(x(t), y(t)) & \leq-2\left(a_{0}-q_{1} L r\right) y^{2}(t) a^{y^{2}(t)}+2|y(t)||\tilde{p}(t)| \\
& =-2\left[\left(a_{0}-q_{1} L r\right)-\frac{|\tilde{p}(t)|}{|y(t)| a^{y^{2}(t)}}\right] y^{2}(t) a^{y^{2}(t)} \\
& \leq-\mu y^{2}(t) a^{y^{2}(t)}
\end{aligned}
$$

for $|y(t)| \geq H_{1}$ and $q(V(x, y(s)))<p(V(x, y(t))), s \in[t-r, t]$. Therefore, the Theorem 1 implies the second coordinate of the solutions of the System (5.2) is uniformly bounded and uniformly ultimately bounded. 


\section{ACKNOWLEDGEMENT}

The author is profoundly grateful to the anonymous reviewer for his help and valuable, profound, constructive comments and suggestions that make us improve the quality of this paper enormously.

\section{REFERENCES}

[1] T. Burton, Stability and Periodic Solutions of Ordinary and Functional Differential Equations. Academic Press, Orlando, 1985.

[2] M. Gu and W. Feng, "Partial boundedness of the impulsive differential equations," Applied Mathematics-A Journal of Chinese Universities (in Chinese), vol. 27, no. 1, pp. 73-83, 2012.

[3] Y. Guo, "Matrix measure and uniform ultimate boundedness with respect to partial variables for FDEs," Journal of Wuhan University of Science and Engineering (in Chinese), vol. 21, no. 4, pp. $15-19,2008$.

[4] J. K. Hale and S. M. V. Lunel, Introduction to Functional Differential Equations. Springer-Verlag, New York, 1993.

[5] T. Hayakawa, W. M. Haddad, N. Hovakimyan, and V. Chellaboina, "Neural network adaptive control for nonlinear nonnegative dynamical systems," IEEE TRANSACTIONS ON NEURAL NETWORKS, vol. 16, no. 2, pp. 399-413, 2005.

[6] X. X. Liao, "Stability, boundedness, dissipation of partial variables for nonlinear systems with separating variables," SCIENCE IN CHINA (Series A), vol. 35, no. 9, pp. 1025-1039, 1992.

[7] X. X. Liao, Y. L. Fu, and Y. X. Guo, "Partial dissipative property for a class of nonlinear systems with separated variables," Journal of Mathematical Analysis and Applications, vol. 173, no. 1, pp. 103-115, 1993.

[8] X. Liao, The mathematical theory and applications of stability(in Chinese). Huazhong Normal University Press, Wuhan, 1988.

[9] X. Lin, "On the boundedness of solutions of difference equations for the partial variables," Journal of Xinjiang University (Natural Science), vol. 7, no. 4, pp. 23-27, 1990.

[10] A. Michel, A. Molchanov, and Y. Sun, "Partial stability and boundedness of discontinuous dynamical systems," Nonlinear Studies, vol. 9, no. 3, pp. 225-247, 2002.

[11] A. Michel, A. Molchanov, and Y. Sun, "Partial stability and boundedness of general dynamical systems on metric spaces," Nonlinear Analysis, vol. 52, pp. 1295-1316, 2003.

[12] B. Shi, D. Zhang, and M. Gai, Theory and Applications of Differential Equations(in Chinese). National Defense Industry Press, Beijing, 2005.

[13] J. Shi, "The theorems for boundedness of solutions of ordinary differential equations with respect to partial variables," Journal of Hanjiang University(Natural Science)(in Chinese), no. no.1, pp. 37-40+47, 1987.

[14] M. Su, "The boundedness about the partial variables of solutions of discrete systems," Journal of Henan Normal University (Natural Science) (in Chinese), no. no.4, pp. 76-79+91, 1990.

[15] M. Wassim, C. VijaySekhar, H. Qing, and H. Tomohisa, "Neural network adaptive control for discrete-time nonlinear nonnegative dynamical systems," Advances in Difference Equations, vol. 2008, no. 9 April 2008, Article ID 868425, pp. 1-29, 2008.

[16] M. Wei, "Partial stability and boundedness of ordinary differential equations under continuous perturbation," Journal of Sichuan Normal University (Natural Science) (in Chinese), vol. 19, no. 3, pp. 29-32, 1996.

[17] H. Xiao, "Boundedness of discrete systems and their perturbed systems with respect to part variables," Journal of Central China Normal University (Natural Science) (in Chinese), vol. 24, no. 2, pp. 143-146, 1990. 
[18] H. Xiao and X. Liao, "Stability and boundedness of large-scale discrete systems with respect to partial variables," Acta Mathematicae Applicatae Sinica (in Chinese), vol. 13, no. 2, pp. 252-256, 1990.

[19] D. Xu and X. Yan, "Boundedness of nonlinear differential equations with respect to part variables," Journal of Sichuan Normal University (Natural Science) (in Chinese), vol. 19, no. 2, pp. 26-32, 1996.

[20] T. Yoshizawa, Stability Theory by Liapunov's Second Method. Math.Soc. Japan, Tokyo, 1966.

[21] J. Zhao, K. Huang, and Q. LU, "Some theorems for a class of dynamical systems with delay and their applications," Acta Mathematicae Applicatae Sinica (in Chinese), vol. 18, no. 3, pp. 422-428, 1995.

Author's address

Jiemin Zhao

Beijing Union University, Department of Mathematics and Physics, 100101 Beijing, China

E-mail address: ldt jiemin@buu.edu.cn 